\title{
ANALYSIS OF RETRIAL FUZZY QUEUE WITH SINGLE SERVER SUBJECT TO BREAKDOWNS AND REPAIRS BY FLEXIBLE ALPHA-CUTS METHOD
}

\section{J. P. MUKEBA KANYINDA}

Department of Mathematics and Computer Science

Higher Institute of Education of Mbuji-Mayi

(ISP Mbuji-Mayi)

Democratic Republic of Congo

e-mail: jpmukeba@yahoo.fr

jeanpierremukebakanyinda2016@gmail.com

\begin{abstract}
This paper introduces the performance measures computation of a single server fuzzy retrial queue with breakdowns and repairs by a new technique called flexible $\alpha$-cuts method. This one uses only one fuzzy arithmetic instead of two arithmetics used by mathematical nonlinear programming method. Basing on this approach, some characteristics as expected customers number in the queue, in the orbit or in the entire system can be computed successfully, and results are the same than those obtained by other methods.
\end{abstract}

2020 Mathematics Subject Classification: 60K20, 03E72.

Keywords and phrases: retrial fuzzy queue, performance measures, breakdowns, repairs, flexible alpha-cuts method.

Received January 2, 2020

(ㄷ) 2020 Scientific Advances Publishers

This work is licensed under the Creative Commons Attribution International License (CC BY 3.0).

http://creativecommons.org/licenses/by/3.0/deed.en_US

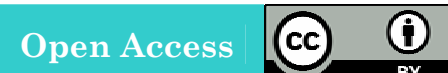




\section{Introduction}

Retrial queues model with breakdowns and repairs is the one of queueing systems which has a wide practical use in manufacturing systems where a server can break down by moment, pass under repair and restart again.

Being given a large number of parameters in this model, its analytical study is usually difficult and addresses only particular cases [8].

A review of main results of the crisp model can be found in [1], [11], [18]. Regarding the fuzzy model, only a few studies investigated this topic. Amoung them, [10] and [17] combined Zadeh's extension principle and mathematical nonlinear programming method to compute performance measures. Using L-R method (Left-Right method) introduced by [15], [12] computed performance measures of the M-M-1 fuzzy model.

The present work uses a new technique introduced recently in [13] and [14], called "flexible $\alpha$-cuts method" to analyse the M-M-1 model in fuzzy environment. Let us recall that this technique consists in applying the alpha-cut and interval arithmetic to fuzzy queueing formulae without resorting to other forms of computation. Its flexibilty and advantage compared to other methods are in the fact that nearly all calculations are nonfuzzy. To show its powerful, we apply it here to queueing model with multimodal fuzzy parameters instead of unimodal fuzzy parameters applied in [13]. Through the illustrative example proposed below, we show that the flexible alpha-cuts method is suitable not only for fuzzy queues having at most three parameters, but also for those contain at most five parameters.

The paper is organized as follows: Section 2 gives some preliminaries needed on fuzzy arithmetic. Section 3 recalls briefly crisp model of retrial queues with breakdowns and repairs. Section 4 proposes the fuzzy model. Section 5 recalls the flexible $\alpha$-cuts method process. To show the validity of the approach, Section 6 proposes a numerical example in which fuzzy customers numbers in the queue, in the orbit and in the entire system are requested at steady state. Section 7 ends the study by a conclusion. 


\section{Preliminaries}

\subsection{Fuzzy set}

Definition 2.1. Let $E$ be a classical set or a universe. A fuzzy subset $\widetilde{A}$ (or a fuzzy set $\tilde{A}$ ) in $E$ is defined by the function $\eta_{\tilde{A}}$, called membership function of $\widetilde{A}$, from $E$ to the real unit interval [0, 1] (see [21]).

In these conditions, $\eta_{\widetilde{A}}(a)$ is called the grade or the membership degree of $a, \forall a \in E$.

Definition 2.2. Let $\widetilde{A}$ be a fuzzy subset in the universe $E$. The alpha-cut $\widetilde{A}_{\alpha}$, the support $\operatorname{supp}(\widetilde{A})$, the height $\operatorname{hgt}(\tilde{A})$, and the core $\operatorname{core}(\tilde{A})$ of $\tilde{A}$, are crisp sets defined respectively as follows $\forall \alpha \in[0,1]$ (see [9]):

$$
\begin{gathered}
\tilde{A}_{\alpha}=\left\{x \in E \mid \eta_{\widetilde{A}}(x) \geq \alpha\right\}, \\
\operatorname{supp}(\tilde{A})=\left\{x \in E \mid \eta_{\widetilde{A}}(x)>0\right\}, \\
\operatorname{hgt}(\tilde{A})=\max \left\{\eta_{\widetilde{A}}(x) \mid x \in E\right\}, \\
\operatorname{core}(\tilde{A})=\left\{x \in E \mid \eta_{\tilde{A}}(x)=1\right\} .
\end{gathered}
$$

The membership function of a fuzzy set $\widetilde{A}$ can be expressed in terms of characteristic functions of its $\alpha$-cuts according to the formula (see [7])

$$
\eta_{\widetilde{A}}(x)=\sup _{\alpha \in[0,1]} \min \left\{\alpha, \eta_{\widetilde{A}_{\alpha}}(x)\right\}
$$

where

$$
\eta_{\tilde{A}_{\alpha}}(x)= \begin{cases}1 & \text { if } x \in \tilde{A}_{\alpha} \\ 0 & \text { otherwise }\end{cases}
$$


Definition 2.3. A fuzzy set $\widetilde{A}$ is said normal if and only if

$$
\operatorname{hgt}(\tilde{A})=1,
$$

and convex if and only if

$$
\eta_{\widetilde{A}}(\lambda x+(1-\lambda y)) \geq \min \left\{\eta_{\widetilde{A}}(x), \eta_{\widetilde{A}}(y)\right\},
$$

$\forall x, y \in E, \forall \alpha \in[0,1]$ (see [19]).

\subsection{Fuzzy number}

Definition 2.4. A fuzzy set $\widetilde{A}$ is said a fuzzy number if $\tilde{A}$ is a fuzzy subset of $\mathbb{R}$ such as (see [3]):

(i) $\operatorname{core}(\widetilde{A}) \neq \emptyset$;

(ii) $\widetilde{A}_{\alpha}$ are all closed and bounded subintervals of $\mathbb{R}$;

(iii) $\operatorname{supp}(\tilde{A})$ is bounded.

Definition 2.5. A fuzzy number $\tilde{A}$ is said strictly positive if $\eta_{\widetilde{A}}(x)=0 \forall x<0$ and strictly negative if $\eta_{\widetilde{A}}(x)=0 \forall x>0$ (see [21]).

Definition 2.6. Let $\widetilde{A}$ and $\widetilde{B}$ be two fuzzy numbers. $\widetilde{A}$ is strictly inferior to $\widetilde{B}(\widetilde{A}<\widetilde{B})$ if and only if $\forall x \in \operatorname{supp}(\widetilde{A}), \forall y \in \operatorname{supp}(\widetilde{B}), x<y$ (see [16]).

In other words,

$$
\widetilde{A}<\widetilde{B} \Leftrightarrow \sup \{\operatorname{supp}(\widetilde{A})\}<\inf \{\operatorname{supp}(\widetilde{B})\} .
$$

Definition 2.7. A fuzzy number $\widetilde{A}$ is said trapezoidal fuzzy number, noted $\tilde{A}=(a / b / c / d)$, if and only if there is four real numbers $a<b<c<d$ such that (see [21]) 


$$
\eta_{\widetilde{A}}(x)=\left\{\begin{array}{ccc}
\frac{x-a}{b-a} & \text { if } & a \leq x \leq b, \\
1 & \text { if } & b<x \leq c, \\
\frac{d-x}{d-c} & \text { if } & c<x \leq d, \\
0 & \text { otherwise. }
\end{array}\right.
$$

Remark 2.1. Trapezoidal fuzzy numbers belong to the set of fuzzy numbers whose the core contains at least two elements, called multimodal fuzzy numbers or flat fuzzy numbers in [5] and [6].

\subsection{Arithmetic on fuzzy numbers}

\subsubsection{Intervals arithmetic}

Definition 2.8. Let $\left[a_{1}, b_{1}\right]$ and $\left[a_{2}, b_{2}\right]$ be two closed, bounded, intervals of real numbers. If $\star$ denotes addition, substraction, multiplication or division, then (see [4])

$$
\left[a_{1}, b_{1}\right] \star\left[a_{2}, b_{2}\right]=[\alpha, \beta]
$$

where

$$
[\alpha, \beta]=\left\{a \star b \mid a_{1} \leq a \leq b_{1}, a_{2} \leq b \leq b_{2}\right\} .
$$

For division, it is assumed that $0 \notin\left[a_{2}, b_{2}\right]$. With basic operations, Equation (8) can be developed as follows:

$$
\begin{aligned}
& {\left[a_{1}, b_{1}\right]+\left[a_{2}, b_{2}\right]=\left[a_{1}+a_{2}, b_{1}+b_{2}\right],} \\
& {\left[a_{1}, b_{1}\right]-\left[a_{2}, b_{2}\right]=\left[a_{1}-b_{2}, b_{1}-a_{2}\right],}
\end{aligned}
$$

$\left[a_{1}, b_{1}\right] \cdot\left[a_{2}, b_{2}\right]=\left[\min \left\{a_{1} a_{2}, a_{1} b_{2}, b_{1} a_{2}, b_{1} b_{2}\right\}, \max \left\{a_{1} a_{2}, a_{1} b_{2}, b_{1} a_{2}, b_{1} b_{2}\right\}\right]$,

$$
\frac{\left[a_{1}, b_{1}\right]}{\left[a_{2}, b_{2}\right]}=\left[\min \left\{\frac{a_{1}}{a_{2}}, \frac{a_{1}}{b_{2}}, \frac{b_{1}}{a_{2}}, \frac{b_{1}}{b_{2}}\right\}, \max \left\{\frac{a_{1}}{a_{2}}, \frac{a_{1}}{b_{2}}, \frac{b_{1}}{a_{2}}, \frac{b_{1}}{b_{2}}\right\}\right] \text {. }
$$




\subsection{2. $\alpha$-Cuts arithmetic}

Let $\widetilde{A}$ and $\widetilde{B}$ be two fuzzy numbers. The fuzzy addition $\oplus$, substraction $\ominus$, multiplication $\odot$ or division $\oslash$ of $\widetilde{A}$ and $\widetilde{B}$ are defined through their $\alpha$-cuts $(0 \leq \alpha \leq 1)$ which are closed and bounded real intervals. If $\widetilde{A}_{\alpha}=\left[A^{L}(\alpha) ; A^{U}(\alpha)\right]$ and $\widetilde{B}_{\alpha}=\left[B^{L}(\alpha), B^{U}(\alpha)\right]$ represent,

respectively the $\alpha$-cuts of $\widetilde{A}$ and the $\alpha$-cuts of $\widetilde{B}$, then [2] and [4] give the basic operations relative to $\alpha$-cuts of $\widetilde{A}$ and $\widetilde{B}$ as follows:

$$
\begin{gathered}
{[\widetilde{A} \oplus \widetilde{B}]_{\alpha}=\widetilde{A}_{\alpha}+\widetilde{B}_{\alpha}=\left[A^{L}(\alpha), A^{U}(\alpha)\right]+\left[B^{L}(\alpha), B^{U}(\alpha)\right],} \\
{[\widetilde{A} \odot \widetilde{B}]_{\alpha}=\widetilde{A}_{\alpha}-\widetilde{B}_{\alpha}=\left[A^{L}(\alpha), A^{U}(\alpha)\right]-\left[B^{L}(\alpha), B^{U}(\alpha)\right],} \\
{[\widetilde{A} \odot \widetilde{B}]_{\alpha}=\widetilde{A}_{\alpha} \cdot \widetilde{B}_{\alpha}=\left[A^{L}(\alpha), A^{U}(\alpha)\right] \cdot\left[B^{L}(\alpha), B^{U}(\alpha)\right],} \\
{[\widetilde{A} \oslash \widetilde{B}]_{\alpha}=\frac{\widetilde{A}_{\alpha}}{\widetilde{B}_{\alpha}}=\frac{\left[A^{L}(\alpha), A^{U}(\alpha)\right]}{\left[B^{L}(\alpha), B^{U}(\alpha)\right]}}
\end{gathered}
$$

The $\alpha$-cuts in Equations (13), (14), (15), and (16) are often computed by using intervals arithmetic formulas in Equations (9), (10), (11), and (12).

\subsection{3. $\alpha$-Cuts and intervals arithmetic}

Definition 2.9. To effect fuzzy arithmetic by " $\alpha$-cuts and intervals arithmetic" consists in using successively

- Equations (13), (14), (15), and (16) for defuzzification.

- Equations (9), (10), (11), and (12) for classical arithmetic on real closed intervals.

- Equation (5) for fuzzification. 


\section{The Crisp Model of Retrial Queue with \\ Breakdowns and Repairs}

\subsection{Description, stability condition and customers number}

Assume that a single class of customers arrives to the queue according to a Poisson process with fuzzy parameter $\tilde{\lambda}$. They form a queue to receive an exponentiel service with fuzzy rate $\tilde{\gamma}$ to the unreliable server whose failure times are independent and exponentially distributed with fuzzy rate $\tilde{\sigma}$. Once a customer is interrupted by server breakdown, he leaves the service area and enters into the retrial orbit

whose rate is a fuzzy number $\widetilde{\theta}$. During this time, the server is assigned in repair whose rate is a fuzzy number $\widetilde{\beta}$. Orbit customers do not rejoin the normal queue but rather attempt to access the server directly after an uncertain amount of time when the server is again operational and iddle. All processes in the system are assumed to be independent and identically distributed. The queue size and the orbit size are assumed to be infinite and the service discipline is FIFO (first in first out).

For such a model, [17] gives the following system stability condition:

$$
\frac{\lambda(\beta+\sigma)}{\beta \gamma}<1
$$

With this previous condition, respective analytical crisp formulas for the customers number in the queue noted $N_{q}$, the customers number in the orbit noted $N_{o}$, and the customers number in the system noted $N_{s}$, are given in [18] and [17] as follows:

$$
\begin{gathered}
N_{q}=\frac{\lambda\left[\gamma \sigma(\gamma+\sigma)+\lambda(\beta+\sigma)^{2}\right]}{\gamma(\beta+\sigma)[\beta(\gamma+\sigma)-\lambda(\beta+\sigma)]}, \\
N_{o}=\frac{\beta \lambda \sigma[\gamma(\gamma+\sigma-\lambda)+\lambda(\beta+\sigma)]}{\gamma[\beta \gamma-\lambda(\beta+\sigma)][\beta(\gamma+\sigma)-\lambda(\beta+\sigma)]}+\frac{\lambda \sigma(\beta+\sigma)}{\theta[\beta \gamma-\lambda(\beta+\sigma)]}, \\
N_{s}=\frac{\lambda\left[\gamma \sigma+(\beta+\sigma)^{2}\right]}{(\beta+\sigma)[\beta \gamma-\lambda(\beta+\sigma)]}+\frac{\lambda \sigma(\beta+\sigma)}{\theta[\beta \gamma-\lambda(\beta+\sigma)]} .
\end{gathered}
$$




\section{The Fuzzy Model}

\subsection{Stability condition in fuzzy model}

Before launching computations in fuzzy model, it is important to verify the queue stability condition. Following the inequalities in (6) and (17), it can easly established that such a fuzzy queue is stable if

$$
\sup \{\operatorname{supp}[\tilde{\lambda}(\tilde{\gamma}+\tilde{\delta})]\}<\inf \{\operatorname{supp}(\tilde{\gamma} \tilde{\mu})\} .
$$

\subsection{Analytical formulae of the fuzzy model}

If the inequality in (21) is verified, we can compute performance measures. On basis of extension principle (see [20], [21], [2]), the crisp performance measures in Equations (18), (19), and (20) can be extended to the following fuzzy measures:

$$
\begin{aligned}
& \tilde{N}_{q}=\frac{\tilde{\lambda}\left[\tilde{\gamma} \widetilde{\sigma}(\tilde{\gamma}+\tilde{\sigma})+\tilde{\lambda}(\tilde{\beta}+\tilde{\sigma})^{2}\right]}{\widetilde{\gamma}(\widetilde{\beta}+\tilde{\sigma})[\tilde{\beta}(\tilde{\gamma}+\tilde{\sigma})-\tilde{\lambda}(\tilde{\beta}+\tilde{\sigma})]}, \\
& \tilde{N}_{o}=\frac{\tilde{\beta} \tilde{\lambda} \widetilde{\sigma}[\tilde{\gamma}(\tilde{\gamma}+\tilde{\sigma}-\tilde{\lambda})+\tilde{\lambda}(\tilde{\beta}+\tilde{\sigma})]}{\tilde{\gamma}[\tilde{\beta} \tilde{\gamma}-\tilde{\lambda}(\tilde{\beta}+\tilde{\sigma})][\tilde{\beta}(\tilde{\gamma}+\tilde{\sigma})-\tilde{\lambda}(\tilde{\beta}+\tilde{\sigma})]}+\frac{\tilde{\lambda} \widetilde{\sigma}(\tilde{\beta}+\tilde{\sigma})}{\tilde{\theta}[\tilde{\beta} \tilde{\gamma}-\tilde{\lambda}(\tilde{\beta}+\tilde{\sigma})]}, \\
& \tilde{N}_{s}=\frac{\tilde{\lambda}\left[\tilde{\gamma} \tilde{\sigma}+(\tilde{\beta}+\tilde{\sigma})^{2}\right]}{(\widetilde{\beta}+\tilde{\sigma})[\tilde{\beta} \tilde{\gamma}-\tilde{\lambda}(\tilde{\beta}+\tilde{\sigma})]}+\frac{\tilde{\lambda} \tilde{\sigma}(\tilde{\beta}+\tilde{\sigma})}{\tilde{\theta}[\tilde{\beta} \tilde{\gamma}-\tilde{\lambda}(\widetilde{\beta}+\tilde{\sigma})]} .
\end{aligned}
$$

\section{Flexible Alpha-Cut Method Process}

Let us precise that the present process can be applied only when analytical queue formulae and input queue parameters are known [13]. Let us suppose that we need to determine a characteristic $\widetilde{\psi}$ of a fuzzy queue whose input parameters are fuzzy numbers $\widetilde{\xi}_{1}, \widetilde{\xi}_{2}, \ldots, \widetilde{\xi}_{n}$. Let us suppose also that $\psi$ and $\xi_{1}, \xi_{2}, \ldots, \xi_{n}$ are respectively the same characteristic and the same parameters in crisp model. If the analytical formula of $\psi$ is known, it is often expressed by

$$
\psi=f\left(\xi_{1}, \xi_{2}, \ldots, \xi_{n}\right),
$$


where $f$ is a real multivalued function using basics operations ",,$+- \times$ and $\div$ " in $\mathbb{R}$. By means of Zadeh's extension principle, the crisp characteristic $\psi$ in Equation (25) is extended to the following fuzzy characteristic:

$$
\widetilde{\psi}=\tilde{f}\left(\widetilde{\xi}_{1}, \widetilde{\xi}_{2}, \ldots, \widetilde{\xi}_{n}\right)
$$

where $\tilde{f}$ is a fuzzy multivalued function using basics fuzzy operations “ $\oplus, \ominus, \odot$ and $\oslash$ ” in the set of fuzzy numbers noted $\mathbb{F}(\mathbb{R})$.

To determine the fuzzy queue characteristic $\widetilde{\phi}$ by flexible $\alpha$-cuts approach, it suffices to determine $\widetilde{\phi}$ by $\alpha$-cuts and intervals arithmetic. That is why we could use the following process:

$\mathbf{1}^{\circ}$ We determine firstly the $\alpha$-cuts of all input parameters $\tilde{\xi}_{1}, \widetilde{\xi}_{2}$, $\ldots, \widetilde{\xi}_{n}$ under the form of closed real intervals to get

$$
\tilde{\xi}_{1_{\alpha}}=\left[\varphi_{1}(\alpha), \omega_{1}(\alpha)\right], \quad \tilde{\xi}_{2_{\alpha}}=\left[\varphi_{2}(\alpha), \omega_{2}(\alpha)\right], \ldots, \quad \tilde{\xi}_{n_{\alpha}}=\left[\varphi_{n}(\alpha), \omega_{n}(\alpha)\right],
$$

where $\varphi_{i}(\alpha)$ and $\omega_{i}(\alpha)$ are usual real-valued functions $(1 \leq i \leq n)$.

$\mathbf{2}^{\circ}$ Applying suitable formulas from Equations (13), (14) (15), and (16) to Equation (26), we obtain

$$
\widetilde{\psi}_{\alpha}=\tilde{f}\left(\widetilde{\xi}_{1_{\alpha}}, \widetilde{\xi}_{2_{\alpha}}, \ldots, \widetilde{\xi}_{n_{\alpha}}\right)=\widetilde{f}\left(\left[\varphi_{1}(\alpha), \omega_{1}(\alpha)\right],\left[\varphi_{2}(\alpha), \omega_{2}(\alpha)\right], \ldots,\left[\varphi_{n}(\alpha), \omega_{n}(\alpha)\right]\right)
$$

$3^{\circ}$ Applying Equations (9), (10) (11), and (12) to Equation (27), we get the closed real interval

$$
\widetilde{\psi}_{\alpha}=\left[\widetilde{\psi}^{L}(\alpha), \widetilde{\psi}^{U}(\alpha)\right],
$$


where $\widetilde{\psi}^{L}(\alpha)$ and $\widetilde{\psi}^{U}(\alpha)$ are usual real-valued functions whose reciprocals define the membership function of $\widetilde{\phi}$ as follows:

$$
\eta_{\widetilde{\psi}}(x)=\left\{\begin{array}{ccc}
\left(\widetilde{\psi}^{L}\right)^{-1}(x) & \text { if } & \widetilde{\psi}^{L}(0) \leq x \leq \widetilde{\psi}^{L}(1) \\
\left(\widetilde{\psi}^{U}\right)^{-1}(x) & \text { if } & \widetilde{\psi}^{U}(1)<x \leq \widetilde{\psi}^{U}(0) \\
0 & \text { otherwise. } &
\end{array}\right.
$$

$4^{\circ}$ The real numbers $\widetilde{\phi}^{L}(0)$ and $\widetilde{\psi}^{U}(0)$ obtained in Equation (28) for $\alpha=0$, fix the support bounds of $\widetilde{\psi}$ and indicate that $\widetilde{\psi}$ is an imprecise real number between $\widetilde{\phi}^{L}(0)$ and $\widetilde{\phi}^{U}(0), \widetilde{\phi}$ does not fall below the lower bound $\widetilde{\psi}^{L}(0)$ or exceed the upper bound $\widetilde{\psi}^{U}(0)$. Its modal value $\widetilde{\psi}^{L}(1)=\widetilde{\psi}^{U}(1)$ is often taken as its most possible value.

In idea to show the validity and the practical use of our approach, the following problem from [17] is solved as a didactic example.

\section{Numerical Example}

\subsection{Problem}

Let us consider a fuzzy queueing system with breakdowns and repairs whose arrivals rate, service rate, retrial rate, failure rate and repair rate are, respectively trapezoïdal fuzzy numbers $\tilde{\lambda}=(3 / 4 / 5 / 6)$, $\tilde{\gamma}=(25 / 26 / 27 / 28), \tilde{\theta}=(14 / 15 / 16 / 17), \tilde{\sigma}=(36 / 37 / 38 / 39), \quad$ and $\widetilde{\beta}=(46 / 47 / 48 / 49)$. How could we proceed to determine the expected customers numbers in the queue, the expected customers numbers in the orbit and the expected customers numbers in the entire system at steady state? 


\subsection{Solution}

We determine the expected customers numbers in the queue, the rest of characteristics are determined similarly.

$\mathbf{1}^{\circ}$ According to Equation (1) in Definition 2.2, let us determine firstly the $\alpha$-cuts of fuzzy parameters $\tilde{\lambda}, \tilde{\gamma}, \widetilde{\theta}, \tilde{\sigma}$, and $\widetilde{\beta}$, we get:

$$
\begin{aligned}
& \tilde{\lambda}_{\alpha}=[\alpha+3,-\alpha+6], \\
& \tilde{\gamma}_{\alpha}=[\alpha+25,-\alpha+28], \\
& \tilde{\theta}_{\alpha}=[\alpha+14,-\alpha+17], \\
& \tilde{\sigma}_{\alpha}=[\alpha+36, \alpha+39], \\
& \tilde{\beta}_{\alpha}=[\alpha+46,-\alpha+49] .
\end{aligned}
$$

$\mathbf{2}^{\circ}$ Applying $\alpha$-cuts arithmetic formula to Equations (22), (23), and (24), we get the results in the following two sub-subsections:

\subsubsection{Expected customers numbers in the queue}

$3^{\circ}$ Let us apply interval arithmetic formulae to Equation (22).

Assume that $\left[\tilde{N}_{q}\right]_{\alpha}=\frac{\widetilde{E}}{\widetilde{F}}$,

with $\widetilde{E}=\tilde{\lambda}_{\alpha}\left[\tilde{\gamma}_{\alpha} \tilde{\sigma}_{\alpha}\left(\tilde{\gamma}_{\alpha}+\tilde{\sigma}_{\alpha}\right)+\tilde{\lambda}_{\alpha}\left(\widetilde{\beta}_{\alpha}+\tilde{\sigma}_{\alpha}\right)^{2}\right]$ and $\widetilde{F}=\tilde{\gamma}_{\alpha}\left(\widetilde{\beta}_{\alpha}+\tilde{\sigma}_{\alpha}\right)$ $\left[\widetilde{\beta}_{\alpha}\left(\tilde{\gamma}_{\alpha}+\tilde{\sigma}_{\alpha}\right)-\tilde{\lambda}_{\alpha}\left(\tilde{\beta}_{\alpha}+\tilde{\sigma}_{\alpha}\right)\right]$.

\section{Computation of the numerator $\widetilde{\boldsymbol{E}}$}

$$
\begin{aligned}
\tilde{\gamma}_{\alpha}+\tilde{\sigma}_{\alpha} & =[\alpha+25,-\alpha+28]+[\alpha+36,-\alpha+39] \\
& =[2 \alpha+61,-2 \alpha+67], \\
\tilde{\beta}_{\alpha}+\tilde{\sigma}_{\alpha} & =[\alpha+46,-\alpha+49]+[\alpha+36,-\alpha+39] \\
& =[2 \alpha+82,-2 \alpha+88],
\end{aligned}
$$




$$
\begin{aligned}
\left(\tilde{\beta}_{\alpha}+\tilde{\sigma}_{\alpha}\right)^{2} & =[2 \alpha+82,-2 \alpha+88]^{2}=\left[(2 \alpha+82)^{2},(-2 \alpha+88)^{2}\right], \\
\tilde{\lambda}_{\alpha}\left(\widetilde{\beta}_{\alpha}+\tilde{\sigma}_{\alpha}\right)^{2} & =[\alpha+3,-\alpha+6]\left[(2 \alpha+82)^{2},(-2 \alpha+88)^{2}\right] \\
& =\left[(\alpha+3)(2 \alpha+82)^{2},(-\alpha+6)(-2 \alpha+88)^{2}\right], \\
\tilde{\gamma}_{\alpha} \cdot \tilde{\sigma}_{\alpha} \quad & =[\alpha+25,-\alpha+28] \cdot[\alpha+36,-\alpha+39] \\
& =[(\alpha+25)(\alpha+36),(-\alpha+28)(-\alpha+39)], \\
\tilde{\gamma}_{\alpha} \cdot \tilde{\sigma}_{\alpha}\left(\tilde{\gamma}_{\alpha}+\tilde{\sigma}_{\alpha}\right) & =[(\alpha+25)(\alpha+36),(-\alpha+28)(-\alpha+39)] \cdot[2 \alpha+61,-2 \alpha+67] \\
= & {[(\alpha+25)(\alpha+36)(2 \alpha+61),(-\alpha+28)(-\alpha+39)(-2 \alpha+67)] . }
\end{aligned}
$$

Let us suppose that $\tilde{K}=\tilde{\gamma}_{\alpha} \cdot \tilde{\sigma}_{\alpha}\left(\tilde{\gamma}_{\alpha}+\tilde{\sigma}_{\alpha}\right)+\tilde{\lambda}_{\alpha}\left(\tilde{\beta}_{\alpha}+\tilde{\sigma}_{\alpha}\right)^{2}$

$$
\begin{aligned}
\widetilde{K}= & {[(\alpha+25)(\alpha+36)(2 \alpha+61),(-\alpha+28)(-\alpha+39)(-2 \alpha+67)] } \\
& +\left[(\alpha+3)(2 \alpha+82)^{2},(-\alpha+6)(-2 \alpha+88)^{2}\right] \\
= & {\left[(\alpha+25)(\alpha+36)(2 \alpha+61)+(\alpha+3)(2 \alpha+82)^{2},(-\alpha+28)(-\alpha+39)\right.} \\
& \left.(-2 \alpha+67)+(-\alpha+6)(-2 \alpha+88)^{2}\right], \\
\widetilde{E}=\tilde{\lambda}_{\alpha} \cdot \widetilde{K} & \\
= & {[\alpha+3,-\alpha+6] .\left[(\alpha+25)(\alpha+36)(2 \alpha+61)+(\alpha+3)(2 \alpha+82)^{2},\right.} \\
& \left.(-\alpha+28)(-\alpha+39)(-2 \alpha+67)+(-\alpha+6)(-2 \alpha+88)^{2}\right] \\
= & {\left[(\alpha+3)\left((\alpha+25)(\alpha+36)(2 \alpha+61)+(\alpha+3)(2 \alpha+82)^{2}\right),\right.} \\
& \left.(-\alpha+6)\left((-\alpha+28)(-\alpha+39)(-2 \alpha+67)+(-\alpha+6)(-2 \alpha+88)^{2}\right)\right] .
\end{aligned}
$$




\section{Computation of the denominator $\widetilde{F}$}

$$
\begin{aligned}
\tilde{\beta}_{\alpha}\left(\tilde{\gamma}_{\alpha}+\tilde{\sigma}_{\alpha}\right) & =[\alpha+46,-\alpha+49][2 \alpha+61,-2 \alpha+67] \\
& =[(\alpha+46)(2 \alpha+61),(-\alpha+49)(-2 \alpha+67)], \\
\tilde{\lambda}_{\alpha}\left(\widetilde{\beta}_{\alpha}+\tilde{\sigma}_{\alpha}\right) & =[\alpha+3,-\alpha+6][2 \alpha+82,-2 \alpha+88] \\
& =[(\alpha+3)(2 \alpha+82),(-\alpha+6)(-2 \alpha+88)], \\
\tilde{\gamma}_{\alpha}\left(\tilde{\beta}_{\alpha}+\tilde{\sigma}_{\alpha}\right) & =[\alpha+25,-\alpha+28][2 \alpha+82,-2 \alpha+88] \\
& =[(\alpha+25)(2 \alpha+82),(-\alpha+28)(-2 \alpha+88)] .
\end{aligned}
$$

Let us suppose that $\tilde{L}=\widetilde{\beta}_{\alpha}\left(\tilde{\gamma}_{\alpha}+\tilde{\sigma}_{\alpha}\right)-\tilde{\lambda}_{\alpha}\left(\tilde{\beta}_{\alpha}+\tilde{\sigma}_{\alpha}\right)$

$$
\begin{aligned}
\widetilde{L}= & {[(\alpha+46)(2 \alpha+61)-(-\alpha+6)(-2 \alpha+88),(-\alpha+49)(-2 \alpha+67)} \\
& -(\alpha+3)(2 \alpha+82)], \\
\widetilde{F}= & \widetilde{\gamma}_{\alpha}\left(\widetilde{\beta}_{\alpha}+\widetilde{\sigma}_{\alpha}\right) \cdot \widetilde{L} \\
= & {[(\alpha+25)(2 \alpha+82),(-\alpha+28)(-2 \alpha+88)] \cdot[(\alpha+46)(2 \alpha+61)} \\
& -(-\alpha+6)(-2 \alpha+88),(-\alpha+49)(-2 \alpha+67)-(\alpha+3)(2 \alpha+82)] \\
= & {[(\alpha+25)(2 \alpha+82)((\alpha+46)(2 \alpha+61)-(-\alpha+6)(-2 \alpha+88)),} \\
& (-\alpha+28)(-2 \alpha+88)((-\alpha+49)(-2 \alpha+67)-(\alpha+3)(2 \alpha+82))] .
\end{aligned}
$$

Using Equation (12), we can write $\left[\tilde{N}_{q}\right]_{\alpha}$ as follows:

$$
\begin{aligned}
& {\left[\tilde{N}_{q}\right]_{\alpha}=\frac{\widetilde{E}}{\widetilde{F}}=\left[\min \left\{\frac{f_{1}(\alpha)}{g_{1}(\alpha)}, \frac{f_{1}(\alpha)}{g_{2}(\alpha)}, \frac{f_{2}(\alpha)}{g_{1}(\alpha)}, \frac{f_{2}(\alpha)}{g_{2}(\alpha)}\right\}\right.} \\
&\left.\max \left\{\frac{f_{1}(\alpha)}{g_{1}(\alpha)}, \frac{f_{1}(\alpha)}{g_{2}(\alpha)}, \frac{f_{2}(\alpha)}{g_{1}(\alpha)}, \frac{f_{2}(\alpha)}{g_{2}(\alpha)}\right\}\right],
\end{aligned}
$$

where $\widetilde{E}=\left[f_{1}(\alpha), f_{2}(\alpha)\right]$ and $\widetilde{F}=\left[g_{1}(\alpha), g_{2}(\alpha)\right]$. 
That is:

$$
\begin{aligned}
& f_{1}(\alpha)=(\alpha+3)\left((\alpha+25)(\alpha+36)(2 \alpha+61)+(\alpha+3)(2 \alpha+82)^{2}\right) \\
& f_{2}(\alpha)=(-\alpha+6)\left((-\alpha+28)(-\alpha+39)(-2 \alpha+67)+(-\alpha+6)(-2 \alpha+88)^{2}\right), \\
& g_{1}(\alpha)=(\alpha+25)(2 \alpha+82)((\alpha+46)(2 \alpha+61)-(-\alpha+6)(-2 \alpha+88)) \\
& g_{2}(\alpha)=(-\alpha+28)(-2 \alpha+88)((-\alpha+49)(-2 \alpha+67)-(\alpha+3)(2 \alpha+82)) .
\end{aligned}
$$

Finally,

$$
\left[\tilde{N}_{q}\right]_{\alpha}=\left[\frac{f_{1}(\alpha)}{g_{2}(\alpha)}, \frac{f_{2}(\alpha)}{g_{1}(\alpha)}\right]
$$

where $f_{1}, f_{2}, f_{3}$, and $f_{4}$ are the functions found above.

\section{$4^{\circ}$ Results and discussion}

Basing on Equation (1), (2), and (4), $\tilde{N}_{q}$ is a fuzzy number whose the support and the core are respectively $\operatorname{supp}\left(\tilde{N}_{q}\right)=\left[\tilde{N}_{q}\right]_{0}=\left[\frac{f_{1}(0)}{g_{2}(0)}, \frac{f_{2}(0)}{g_{1}(0)}\right]$ $=] 0.03,0.153\left[\right.$ and $\operatorname{core}\left(\tilde{N}_{q}\right)=\left[\tilde{N}_{q}\right]_{1}=\left[\frac{f_{1}(1)}{g_{2}(1)}, \frac{f_{2}(1)}{g_{1}(1)}\right]=[0.0549,0.0937]$.

These results mean that the customers number in queue belongs to the interval ]0.03, 0.153[. Its most possible value belongs to the interval [0.0549, 0.0937]. According to Equation (30), the following membership function graphic of $\tilde{N}_{q}$, presented in Figure 1 below, is obtained by the following Latex program when some figures packages as Graphicx, Tikz, $P g f$ have been carefully declared in the preamble.

\section{Program}

$\backslash$ begin\{figure $[$ !ht $]$

$\backslash$ begin $\{$ center $\}$

$\backslash$ begin $\{$ tikzpicture $\}[$ xscale $=40$, yscale $=3]$ 
$\backslash$ draw $[->](0,0)-(0,1.3)$ node[left] $\{\$ \backslash$ alpha $\$\}$;

$\backslash$ draw $[->](0,0)-(0.2,0)$ node[above] $\left\{\$ \backslash\right.$ tilde $\left.\{\mathrm{N}\} \_\mathrm{q} \$\right\}$;

$\backslash$ draw [ thick, blue] $(0.0549,1)-(0.096,1)$;

$\backslash$ draw [ ] $(0.03,0)$ node[below] \{ $\backslash$ scriptsize $\backslash$ textcolor\{blue $\{\{0.03\}\}\}$;

$\backslash$ draw [ ] $(0.0549,0)$ node[below] $\backslash$ scriptsize $\backslash$ textcolor $\{$ blue $\}\{0.0549\}\}$;

$\backslash$ draw [ ] $(0.0937,0)$ node[below] $\backslash \backslash$ scriptsize\{ $\backslash$ textcolor $\{$ blue $\}\{0.0937\}\}\}$;

$\backslash$ draw[ ] $(0.153,0)$ node[below] \scriptsize $\backslash$ textcolor\{blue $\{0.153\}\}\}$;

$\backslash$ draw [ dotted] $(0.0549,1)-(0.0549,0)$;

$\backslash$ draw [ dotted] $(0.0937,1)-(0.0937,0)$;

$\backslash$ draw[ ] $(0,0)$ node[left] $\backslash$ scriptsize $\$ textcolor\{blue $\{0\}\}\} ;$

$\backslash$ draw[ ] $(0,1)$ node[left] $\backslash$ scriptsize $\{$ textcolor\{blue $\}\{1\}\}\} ;$

$\backslash$ draw $[$ dotted] $(0.0549,1)-(0,1)$;

$\backslash$ draw[ ] $(0.0773,1)$ node[above] $\bigwedge$ small $\backslash$ textcolor $\{$ blue $\}\left\{\$\{\text { eta }\}_{-} \bigwedge\right.$ tilde $\left.\left.\left.\{\mathrm{N}\}_{-} \mathrm{q}\right\} \$\right\}\right\}$;

$\backslash$ draw [ thick, blue, domain $=0: 1]$ plot $\left(\left\{\left((\backslash \mathrm{x}+3) *\left(0.1^{*} \backslash \mathrm{x}+2.5\right)^{*}\left(0.1^{*} \backslash \mathrm{x}+3.6\right)\right.\right.\right.$

$\left.\left.*\left(0.2^{*} \backslash \mathrm{x}+6.1\right)\right)+\left((\backslash \mathrm{x}+3) *\left(0.1^{*} \backslash \mathrm{x}+0.3\right) *\left(0.2^{*} \backslash \mathrm{x}+8.2\right) *\left(0.2^{*} \backslash \mathrm{x}+8.2\right)\right)\right) /(((-\backslash \mathrm{x}+28)$

$\left.*\left(-0.2^{*} \backslash x+8.8\right) *\left(-0.1^{*} \backslash x+4.9\right) *\left(-0.2^{*} \backslash x+6.7\right)\right)-\left(\left(-0.1^{*} \backslash x+2.8\right)^{*}\left(-0.2^{*} \backslash x+8.8\right)^{*}(\backslash x+3)\right.$

$\left.\left.\left.\left.*\left(0.2^{*} \backslash \mathrm{x}+8.2\right)\right)\right)\right\}, \backslash \mathrm{x}\right)$;

$\backslash$ draw [ thick, blue, domain $=0: 1]$ plot $\left(\left\{\left(\left(\left(-1^{*} \backslash x+6\right)^{*}\left(-0.1^{*} \backslash x+2.8\right)^{*}\left(-0.1^{*} \backslash x+3.9\right)\right.\right.\right.\right.$ $\left.\left.*\left(-0.2^{*} \backslash \mathrm{x}+6.7\right)\right)+\left((-\backslash \mathrm{x}+6)^{*}\left(-0.1^{*} \backslash \mathrm{x}+0.6\right)^{*}\left(-0.2^{*} \backslash \mathrm{x}+8.8\right)^{*}\left(-0.2^{*} \backslash \mathrm{x}+8.8\right)\right)\right) /(((\backslash \mathrm{x}+25)$ $\left.*\left(0.2^{*} \backslash \mathrm{x}+8.2\right)^{*}\left(0.1^{*} \backslash \mathrm{x}+4.6\right)^{*}\left(0.2^{*} \backslash \mathrm{x}+6.1\right)\right)-\left((\mathrm{x}+25)^{*}\left(0.2^{*} \backslash \mathrm{x}+8.2\right)^{*}\left(-0.1^{*} \backslash \mathrm{x}+0.6\right)\right.$ $\left.\left.\left.\left.*\left(-0.2^{*} \backslash \mathrm{x}+8.8\right)\right)\right)\right\}, \backslash \mathrm{x}\right)$;

$\backslash$ end $\{$ tikzpicture\}

$\backslash$ caption\{Membership function of expected customers number in the queue\}

$\backslash$ label\{fig1\}

$\backslash$ end\{center\}

$\backslash$ end\{figure\} 


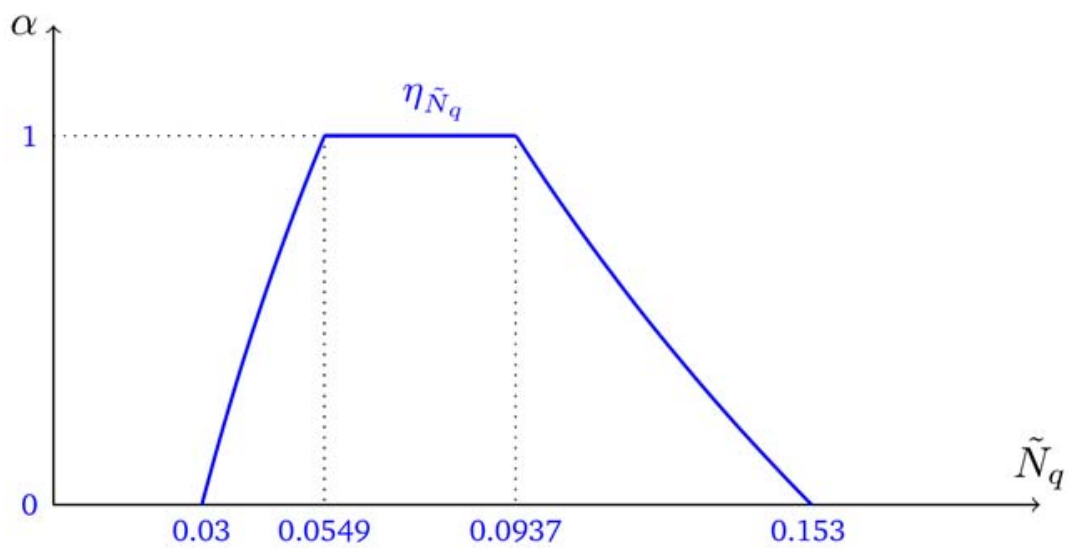

Figure 1. Membership function of expected customers number in the queue.

\subsubsection{Expected customers number in the orbit and expected customers number in the system}

The expected customers number in the orbit and the customers number in the entire system can be found in the analogous manner.

\section{Conclusion}

In the fuzzy queueing theory literature, the retrial model with breakdowns and repairs have been studied less and less further to a large number of fuzzy parameters. Out of the nonlinear programming method and the left-right method already introduced to compute its characteristics, the present article reveals that the flexible alpha-cut method is also suitable to deal with this model as shown through the example proposed in the preceeding section. In this example, the expected customers number in the queue is successfully computed and the result is obtained in fuzzy environment. This method conducts to the same results than those obtained by the other methods and enriches from then on the queueing theory literature. Compared to these other methods, this one seems flexible because all calculations used in are 
nonfuzzy. That is why it is appropriate to fuzzy queueing models containing a large number of parameters. In the next time, we shall investigate if it can solve easily the non-Markovian fuzzy queueing models.

\section{References}

[1] A. Aissani, On the $M / G / 1 / 1$ queueing system with repeated orders and unreliable server, Journal of Technology 6 (1988), 88-123 (in French).

[2] B. Bede, Mathematics of Fuzzy Sets and Fuzzy Logic, Springer-Verlag, Berlin, 2013.

DOI: http://dx.doi.org/10.1007/978-3-642-35221-8

[3] J. J. Buckley and E. Eslami, An Introduction to Fuzzy Logic and Fuzzy Sets, Springer-Verlag, Berlin, Heidelberg, 2002.

DOI: http://dx.doi.org/10.1007/978-3-7908-1799-7

[4] J. J. Buckley, E. Eslami and T. Feuring, Fuzzy Mathematics in Economics and Engineering, Physica-Verlag, Heidelberg, 2010.

[5] D. Dubois and H. Prade, Operations on fuzzy numbers, International Journal of Systems Science 9(6) (1978), 613-626.

DOI: https://doi.org/10.1080/00207727808941724

[6] D. Dubois and H. Prade, Fuzzy real algebra: Some results, Fuzzy Sets and Systems 2(4) (1979), 327-348.

DOI: https://doi.org/10.1016/0165-0114(79)90005-8

[7] D. Dubois and H. Prade, Fuzzy Sets and Systems: Theory and Applications, Academic Press, New York, 1980.

[8] I. Florea and C.-S. Nanau, An algorithmic approach of retrial queuing system with one serving station, Part I: The description of the simulation algorithm, Bulletin of the Transilvania University of Brasov; Série III: Mathematics Informatics Physics 6(2) (2013), 95-106.

[9] M. Hanss, Applied Fuzzy Arithmetic: An Introduction with Engineering Applications, Springer-Verlag, Berlin, Heidelberg, 2005.

DOI: https://doi.org/10.1007/b138914

[10] R. Kalayanaraman, N. Thillaigovindan and G. Kannadasan, A single server fuzzy queue with unreliable server, International Journal of Computational Cognition 8(1) (2010), 1-4.

[11] V. G. Kulkarni and B. D. Choi, Retrial queues with server subject to breakdowns and repairs, Queueing System 7(2) (1990), 191-208.

DOI: https://doi.org/10.1007/BF01158474 
[12] Z. Mueen, R. Ramli and N. Zura Zaibidi, Performance measurements of single server fuzzy queues with unreliable server using left and right method, AIP Conference Proceedings 1691(14) (2015); Article 030019.

DOI: https://doi.org/10.1063/1.4937038

[13] J. P. Mukeba Kanyinda, Analysis of fuzzy queue characteristics by flexible alphacuts method, Journal of Fuzzy Sets Valued Analysis 2017(1) (2017), 1-11.

DOI: http://dx.doi.org/10.5899/2017/jfsva-00344

[14] J. P. Mukeba Kanyinda, Application of L-R fuzzy arithmetic to real-word problems, Journal of Fuzzy Sets Valued Analysis 2019(1) (2019), 1-13.

DOI: http://dx.doi.org/10.5899/2019/jfsva-00463

[15] J. P. Mukeba Kanyinda, R. Mabela Makengo Matendo and B. Ulungu Ekunda Lukata, Computing fuzzy queueing performance measures by L-R method, Journal of Fuzzy Sets Valued Analysis 2015(1) (2015), 57-67.

DOI: http://dx.doi.org/10.5899/2015/jfsva-00226

[16] J. P. Mukeba Kanyinda, R. Mabela Makengo Matendo and B. Ulungu Ekunda Lukata, Performance measures of a fuzzy product form queueing network, Journal of Fuzzy Sets Valued Analysis 2015(1) (2015), 68-77.

DOI: http://dx.doi.org/10.5899/2015/jfsva-00228

[17] S. Shanmugasundaram and B. Venkatesh, An M/M/1 retrial queue with unreliable server under fuzzy environment, International Journal of Mathematical Sciences \& Applications 6(1) (2016), 81-88.

[18] N. P. Sherman and J. P. Kharoufeh, An M/M/1 retrial queue with unreliable server, Operational Research Letters 34(6) (2006), 697-705.

DOI: https://doi.org/10.1016/j.orl.2005.11.003

[19] L. A. Zadeh, Fuzzy sets, Information and Control 8(3) (1965), 338-353.

DOI: http://dx.doi.org/10.1016/S0019-9958(65)90241-X

[20] L. A. Zadeh, Fuzzy sets as a basis for a theory of possibility, Fuzzy Sets and Systems 1(1) (1978), 3-28.

DOI: https://doi.org/10.1016/0165-0114(78)90029-5

[21] H.-J. Zimmermann, Fuzzy Set Theory -and its Applications, Springer Science + Business Media, New-York, Fourth Edition, 2001.

DOI: http://dx.doi.org/10.1007/978-94-010-0646-0 